\title{
EDITORIAL: Paths to adulthood and advances in
}

\section{anonymisation}

\author{
Heather Joshi
}

This last issue of Volume 9 has cross-cutting contributions from a number of countries in Europe - including (still) the UK - and North America, and from sociology, demography, epidemiology and statistics. The empirical research is mainly confined to people in early to mid adulthood from age 18 to 40. Readers interested in childhood or later life may nevertheless find the articles of relevance. The paper on data privacy addresses a generally important question. This introduction tries to bring out some common themes.

The opening paper uses longitudinal data to address an important issue in the study of the lifecourse. In $A$ cohort analysis of subjective wellbeing and ageing: heading towards a midlife crisis?, Steffen Otterbach, Alfonso Sousa-Poza and Valerie Møller set out an account of the ongoing debate about the course of human happiness as individuals pass through the lifecycle. Some argue that the profile is U-shaped with a 'natural' dip in wellbeing at some point in the middle. This not only conforms to the notion of a 'mid-life crisis' but is also claimed to be observed in great apes. Much existing evidence is based on cross-sections, which may also reflect differences between cohorts rather than the effect of the passage of time on an individual. Otterbach and colleagues contribute longitudinal evidence on individuals followed annually for eight years from 2008 to 2015 in Germany. They are from a sequence of three nearoverlapping cohorts each born during three years at the start of the 1970s, 1980s and 1990s respectively. They comprise the German Family Panel, otherwise known as pairfam (yes, no capital letters!). The cohorts provide eight observations running forwards across ages roughly 16-23, 26-33 and 36-43. They report on overall life satisfaction and satisfaction with various aspects of life at each year. There is no evidence of life beyond 44 in the dataset so far, but it does provide substantial evidence for the trajectories of the average levels of satisfaction, among these cohorts, over the years in question. These trajectories are presented in three versions: as raw averages, adjusted for personal and macro-economic circumstances, and as estimates abstracting from unobserved personal attributes via fixed effects regressions. All versions are reasonably close. So are the trajectories for males and females. If the satisfaction variables are strung out from 16 to 43 , it could be argued that at the evidence is generally consistent with a downward slope on the left-hand side of a life-time $U$ shape. This needs to be qualified by the caveat that the different types of satisfaction show very differently shaped pathways towards the hypothetical mid-life milestone. Most of the decline in overall satisfaction comes between teens and twenties, with only a gentle downward slope within (and between) the cohorts followed from mid-twenties and mid-thirties. There is a similar general pattern for satisfaction with leisure activities and social life. Satisfaction with family life does drop off for all of the age groups over the years of follow-up, providing the strongest evidence for a 'nadir' around 40. Satisfaction from 'school, work or career' shows an opposite trend for the average German person in all three cohorts. This suggests that this field of research in other countries, and indeed in the attempts of official statistical agencies to chart wellbeing, should look into the various facets of life satisfaction.

This superficial account of the findings ignores the discontinuity of the profiles where the oldest year of one cohort jumps to the youngest year of the next. Cohort as well as age differences need to be understood. As the authors discuss, while individuals grow older, time inevitably proceeds. The concept of the life course admits the inextricability of the process of individuals growing up or growing old from the march of historical time. This paper brings a lot of thought, as well as evidence, to the question posed in its title, but it is only the beginning of an answer.

Our second paper, by Juli Simon Thomas, Health effects of work and family transitions also looks at mid-life. The focus is on self-reported health and depressive symptoms, at age 40 , as they may be predicted by earlier life events. Events under scrutiny are those involving the gain or loss of a job 
or of a partner, and their timing and coincidence. These mid-life outcomes and their predictors are measured in the American cohort born in 19571964, the NLSY79. The outcomes are similar to, but distinct from, the subjective wellbeing studied by Otterbach and colleagues. The life events, especially when divorce and job loss coincide, might be experienced as crises, but they may take place at any point from 18 to 40 , not necessarily at 'midlife'. While life events are adjusted-out of two versions of the German age-trajectories, here their importance as triggers of poor health at 40 is brought to the fore. In this approach, differences between men and women are more evident than in the life satisfaction results from Germany. Job loss and divorce have stronger negative associations with health at 40 than the positive associations with marriage and employment entry. Marriage has a stronger 'protective effect' against depression for women than men. Although the more recent events show greater impact, events before age 26 show persistent traces. The main message is that the effects of transitions are 'riddled with intersectionalities'; troubles tend to come together. This complements other work by this author on transitions in life domains in USA and their outcomes. Her study of families in the Panel Study of Income Dynamics, Dimensions of family disruption: coincidence and impacts on children's attainments (published in Longitudinal and Life Course Studies, 2018, Vol 9, no 2, pp.157-187), also includes residential mobility alongside partnership and employment transitions as predicting high school and college attainments of the adults' offspring.

The third article in this number addresses a completely different but also very important issue in research on longitudinal data, the preservation of informant privacy. Although this feature of data management is usually taken for granted, breaches of confidentiality have the potential for a crisis, not only for the dataset in which they occur, but in damaging public confidence in the basis for giving information to surveys, longitudinal surveys in particular. Demetris Avraam, Andy Boyd, Harvey Goldstein and Paul Burton summarise their contribution in their title: A software package for the application of probabilistic anonymisation to sensitive individual-level data: a proof of principle with an example from the ALSPAC birth cohort study. Recognising the need to balance disclosure control with retaining data utility, this team have combined expertise in data management, epidemiology and statistics to develop a tool for data custodians of longitudinal studies. It builds on an existing practice of perturbing data to make it safe to release, but gives analysts enough information to allow for the artificial errors introduced into the file anonymised for secondary analysis. They show worked examples on a dataset about asthma in this proof of principle exercise. The fruition of this project should help longitudinal studies 'to maintain participant trust and to share data securely and effectively while meeting ever more stringent data protection requirements'.

The notion of resilience to stress appears in the first article as one theory for why the life satisfaction may recover, if it does, after a mid-life nadir. It takes centre stage in the research note on national register data from Sweden by Scott Montgomery and colleagues, Sex of older siblings and stress resilience. Their evidence comes from a rigorous assessment administered to young men in Sweden facing compulsory military service as to whether they had the capability to cope with the stress of combat. This is by far better quality data on psychological resilience than can normally be collected in multi-purpose surveys. It is thus of interest even though it does not extend much beyond age 20 or to young women, or beyond Sweden. The question addressed in this research note is how the assessed ability to cope under stress is related to antecedent family background, in particular the presence of siblings and their sex, for which there is also evidence in linked registers. The authors add, to the (not unmixed) literature on the advantage of being first-born, the finding that older brothers present more of a challenge than older sisters. To put it much more crudely than the authors, 'Who's afraid of a big, bad brother?' On another note, I noticed another point in common with Otterbach et al.: in both papers the useful literature reviews include animal studies.

The potential to study relationships among life events, and between them and wellbeing outcomes, is one of many applications offered by the Gender and Generations Survey featured in this issue's study profile, by Anne Gauthier, Susana Cabaço and Tim Edery from the Netherlands Interdisciplinary Demographic Institute. It is part of the Gender and Generations Programme funded by the European Union. This valuable component of 
the European longitudinal data infrastructure runs the GGS surveys in 21 countries (in east and west Europe) as well as hosting harmonised histories on childbearing and marital events from UK, USA, and Uruguay. The GGS surveys start in these countries and at dates mostly in the first decade of the 21st century (varying by country) and following individuals, initially aged $18-79$, at three-year intervals. They hence represent birth cohorts from the last three-quarters of the 20th century. The study profile concentrates on those countries where there are two waves of data already available to external researchers. A new round of surveys is expected to begin in 2020. The focus of data collection, and analysis to date, has been on family dynamics, ranging across the life course from leaving home, through childbearing to care of the elderly. It is a resource of growing potential for internationally comparative research and information in our field. Readers of this journal who are not yet familiar with it are recommended to take a look, and to consider making use of it. 